This item was submitted to Loughborough's Research Repository by the author.

Items in Figshare are protected by copyright, with all rights reserved, unless otherwise indicated.

\title{
The European road safety decision support system on risks and measures
}

\section{PLEASE CITE THE PUBLISHED VERSION}

https://doi.org/10.1016/j.aap.2018.08.005

\section{PUBLISHER}

(C) Elsevier

\section{VERSION}

AM (Accepted Manuscript)

\section{PUBLISHER STATEMENT}

This paper was accepted for publication in the journal Accident Analysis and Prevention and the definitive published version is available at https://doi.org/10.1016/j.aap.2018.08.005.

\section{LICENCE}

CC BY-NC-ND 4.0

\section{REPOSITORY RECORD}

Martensen, Heike, Kevin Diependale, S. Daniels, W. Van den Berghe, Eleonora Papdimitriou, George Yannis, Ingrid van Schagen, et al.. 2018. "The European Road Safety Decision Support System on Risks and Measures”. Loughborough University. https://hdl.handle.net/2134/34490. 


\title{
The European Road Safety Decision Support System on Risks and Measures
}

\author{
Heike Martensen'; Kevin Diependaele; Stijn Daniels'; Wouter Van den Berghe'; Eleonora \\ Papadimitriou'; George Yannis²; Ingrid Van Schagen $^{3}$; Wendy Weijermars 3 ; Wim Wijnen ${ }^{4}$; Ashleigh \\ Filtness $^{5}$; Rachel Talbot ${ }^{5}$; Pete Thomas ${ }^{5}$; Klaus Machata ${ }^{6}$; Eva Aigner Breuss ${ }^{6}$; Susanne Kaiser ${ }^{6}$; Thierry \\ Hermitte $^{7}$; Rob Thomson ${ }^{8}$; Rune Elvik ${ }^{9}$
}

1 Vias Institute, Belgium; 2 Department of Transportation Planning and Engineering, NTUA, Greece; 3 Institute for Road Safety Research, SWOV, The Netherlands, 4 W2 Economics, The Netherlands; 5 Loughborough Design School, United Kingdom; 5 Kuratorium für Verkehrssicherheit (KfV), Austria; 7 LAB, PSA Peugeot Citroën, France; 8 SAFER, Chalmers University, Sweden; 9 Institute for Transport Economics (TOI), Norway.

Corresponding author: Dr. Heike Martensen, Vias institute, Haachtsesteenweg 1405, 1130 Brussels, heike.martensen@vias.be

\begin{abstract}
The European Road Safety Decision Support System (roadsafety-dss.eu) is an innovative system providing the available evidence on a broad range of road risks and possible countermeasures. This paper describes the scientific basis of the DSS. The structure underlying the DSS consists of (1) a taxonomy identifying risk factors and measures and linking them to each other, (2) a repository of studies, and (3) synopses summarizing the effects estimated in the literature for each risk factor and measure, and (4) an economic efficiency evaluation instrument (E3-calculator). The DSS is implemented in a modern web-based tool with a highly ergonomic interface, allowing users to get a quick overview or go deeper into the results of single studies according to their own needs.
\end{abstract}

\section{Keywords}

Road safety countermeasures, road risks, effectiveness, online system, review, cost-benefit

\section{Acknowledgement}

The roadsafety-dss has been implemented in the EU research project SafetyCube, funded by the European Commission. Grant agreement No 633485, H2020-MG-2014-2015

\section{Introduction}

The European Road Safety Decision Support System (DSS) was implemented by the research project SafetyCube (Safety CaUsation, Benefits and Efficiency), a European Commission supported Horizon 2020 project with the objective of developing an innovative system that enables policy-makers and stakeholders to select and implement the most appropriate strategies, measures and cost-effective approaches to reduce casualties of all road user types and all severities. The DSS can be found at https://www.roadsafety-dss.eu.The core of system is formed by comprehensive analyses of a broad range of accident risks and the effectiveness of safety measures. The risks and measures investigated cover the areas road users, infrastructure, vehicles and post impact care and are framed within a system approach. Road safety stakeholders at the local, regional and national level, as well as the EU level and beyond have been consulted at all stages of the project.

The functionalities of the system combine the positive aspects of a number of other information sources that functioned as role models for the DSS. The most important role model is the "Handbook of Road Safety Measures” (Elvik, Hoye, Vaa, \& Sorensen; 2009) which summarizes the knowledge from different countries on countermeasures directed towards infrastructure, vehicles, human behavior and post impact care. It also contains an overview of factors contributing to road crashes. Another important role model is the Crash Modification Factor clearinghouse (NHTSA, www.cmfclearinghouse.org), an online repository in which users can find some 6000 estimates of crash-modification factors for a broad range of infrastructure measures. The NHTSA also offers a guideline on countermeasures addressing road-user behavior in their 400 pages volume "Countermeasures that work" a broad range of behavioral risks are described followed by a number of possible countermeasures to address them. For each countermeasure there is a description and a number of implementation examples (Goodwin, Thomas, Kirley, Hall, O’Brien, \& Hill, 2015). Both NHTSA sources are restricted to American case studies. The iRAP toolkit (http://toolkit.irap.org/) gives very nice and compact overview on 58 countermeasures - mainly on infrastructure. The tool also includes implementation examples from different countries. However, the link to effect estimates and other scientific background knowledge is difficult to make. 
The objective of the present DSS was to combine the advantages of each of these systems:

- The scientific basis: a repository of single studies and effects

- Summaries of the resulting knowledge

- A broad focus on human behavior, infrastructure, vehicles, as well as post-crash factors

- A careful analysis of the risks addressed by the countermeasures

- Accessible in a user-friendly online system

Moreover, there is a large body of research on risk-factors to road crashes, and while some of the volumes on counter-measures include summaries of these results as well, there is no systematic one-to-one link between risk factors and counter measures available. Such a linking is important to guide the user's attention to the whole driving system. As an example, driving when tired is generally considered a behavioral risk. However, it can be countered with typical "behavioral measures" like sensitization campaigns or fitness to drive assessment (in the case of sleep apnea) but also with infrastructure measures like rumble strips or the creation of obstacle free spaces as well as vehicle measures like lane departure warning or drowsiness recognition. These links can only be provided in a dynamic online system that spans road safety measures from all domains of the driving system.

\section{The DSS methodology}

The DSS is a dynamic online tool and its underlying structure consists of (1) a taxonomy identifying risk factors and measures and linking them to each other, (2) a repository of studies, and (3) synopses summarizing the effects estimated in the literature for each risk factor and measure, and (4) an economic efficiency evaluation instrument (e3-calculator).

The taxonomy consists of four parts; (1) Road Users, (2) Infrastructure, (3) Vehicles and (4) Post Impact Care. The taxonomy is a main structural part of the DSS, it can be used as a search option in the DSS, it creates a uniform structure over all domains and forms the basis for linking risk factors with their corresponding measures. The structure consists of three levels, which are topic, subtopic and specific topic.

The repository is a data-base of coded studies. The most important challenge for coding studies for the repository is the big variety of topics addressed, which also means that the studies addressing the topics tend to have different designs. A flexible coding template has been developed to be able to include different kinds of quantitative evaluation studies, preserving the information about study-design and type of information collected, but also allowing comparison of the results.

On the basis of the studies coded in the repository, a synopsis is written for each risk factor and each countermeasure summarising the known effects of risk factors or measures by means of meta-analysis, votecount analysis, or simply an overview. To address different types of DSS-users, each synopsis consists of three parts: (1) Summary, (2) Scientific overview, (3) Supporting document.

The DSS is implemented in a modern web-based tool with an ergonomic interface. The structure is determined by the taxonomy and is complemented by a powerful search engine. The DSS allows users to find synopses or single studies related to one or more risk factors or measures, to define their own search criteria (e.g. only studies from a particular country), to identify measures most appropriate to address a particular risk factor, and to have a quick overview of the "riskiness" of risk factors or the effectiveness of measures.

This paper discusses the four core elements of the road safety DSS in more detail and describes the scientific basis of the DSS.

\subsection{Taxonomy}

The risk factors and the measures to be included in the DSS were identified based on a systematic analysis of the road safety literature. The risks and measures were assigned to one of four main areas:

- $\quad$ Road users

- Infrastructure

- Vehicles

- $\quad$ Post impact care (only measures)

The draft taxonomy was systematically evaluated during four workshops, where stakeholders were asked to prioritize and indicate missing topics. Three workshops were directed to a general audience of road safety policy makers and practitioners, one was focused on infrastructure (Filtness, et al, 2016).

For each of the risk and measures areas, a three-level taxonomy was developed consisting of the main topic at level 1 , several subtopics at level 2, and, if appropriate, specific topics at level 3. Table 1 shows all first level 
topics addressed in the DSS. For each of the main topics presented in Table 1, there is a hierarchy of sub-topics and specific topics. To present the full hierarchy here, would exceed the space for this paper. As an example, Table 2 presents the sub-topics and specific topics related to "speed choice" and "fatigue", two of the main topics from the road user area.

Table 1: Level 1 topics of the taxonomy

\begin{tabular}{|c|c|c|}
\hline & Risk Factor & Measure \\
\hline \multirow[t]{14}{*}{ Road Users } & Speed Choice & Law and Enforcement \\
\hline & Influenced Driving - alcohol & Education and Voluntary Training/programs \\
\hline & Influenced Driving - drugs & Driver Training and Licensing \\
\hline & Risk Taking & Fitness to Drive Assessment and Rehabilitation \\
\hline & Fatigue & Awareness Raising and Campaigns \\
\hline & Distraction and Inattention & \\
\hline & Functional Impairment & \\
\hline & Insufficient Knowledge & \\
\hline & Emotion and Stress & \\
\hline & Misjudgement and Observation Errors & \\
\hline & Traffic Rule Violations & \\
\hline & Personal Factors & \\
\hline & Age & \\
\hline & Disease and Disorders & \\
\hline \multirow[t]{11}{*}{ Infrastructure } & Exposure & Exposure \\
\hline & Road Type & Infrastructure Safety Management \\
\hline & Road Surface & Road Type \\
\hline & Road Environment & Road Surface \\
\hline & Workzones & Lighting \\
\hline & Alignment Deficiencies - Road Segments & Workzones \\
\hline & Cross-section Deficiencies - Road Segments & Alignment - Road Segments \\
\hline & Traffic control - Road Segments & Cross-section - Road Segments \\
\hline & Alignment - Junctions & Traffic Control - Road Segments \\
\hline & Traffic Control - Junctions & Alignment - Junctions \\
\hline & & Traffic Control - Junctions \\
\hline \multirow[t]{7}{*}{ Vehicles } & Crashworthiness & Crashworthiness \\
\hline & Injury Mechanism & Active Safety/ADAS \\
\hline & Protective Equipment Design & Tertiary Safety \\
\hline & Relevant Factors in Crash Data & \\
\hline & Technical Defects/Maintenance & \\
\hline & Vehicle Design & \\
\hline & Visibility/Conspicuity & \\
\hline \multirow[t]{5}{*}{ Post impact care } & - & Ambulances/Helicopters \\
\hline & & Extraction from Vehicle \\
\hline & & Pre-hospital Medical Care \\
\hline & & Triage and Allocation to Trauma Facilities \\
\hline & & First Aid Training for Drivers \\
\hline
\end{tabular}

Table 2: An Example of the taxonomy (Road users)

\begin{tabular}{ccc}
\hline Level 1 - main topic & Level 2 - subtopic & Level 3 - specific topic \\
\hline Speed choice & Excess speed & Built-up areas \\
\cline { 2 - 2 } & & Rural roads \\
\cline { 2 - 3 } & & Motorways \\
\cline { 2 - 3 } & & Too fast weather-related \\
\cline { 2 - 2 } Fatigue & Insufficient (good) & Too fast traffic related \\
\cline { 2 - 2 } & sleep & Too slow \\
\cline { 2 - 3 } & Long drives & Sleeping disorders \\
\cline { 2 - 2 }
\end{tabular}

All risks in the SafetyCube DSS taxonomies are linked to measures that have the potential of reducing this risk. This means a measure (e.g. winter maintenance) is linked to a risk-factor (e.g. snow), even for cases for which it may turn out not to be effective. The idea behind this is to give users access to existing evaluations of the measure, whenever they might consider the measure to be a solution to their problem. 
The links between risks and measures are based on a dedicated SafetyCube model (Yannis \& Papadimitriou, 2018) differentiating between (a) generic risks concerning the general state of the system (e.g., design of roads or vehicles, knowledge of the road users, etc.) and (b) circumstantial risks concerning the transient state of the system at the moment the crash occurred (e.g. defects, environmental conditions, road-user impairment, etc.). The measures were categorized into the same categories depending on the risk factors they mainly address. Moreover, risks and measures are checked for association with specific accident categories (e.g. head-on crashes, or rear-end crashes) and specific accident phases (pre-crash, crash, and post-crash, Haddon, 1980). All these elements are integrated and taken into account when checking for measures that should be considered as remedies for a risk factor in question. Moreover, by linking risk factors to measures from different domains, a systems approach is emphasized for the user. As an example, when looking for measures linked to a road user related risk like "speeding", the user is guided to measures that address road users (campaigns, demerit point systems) or infrastructure (speed humps, section control) or the vehicle (ISA, adaptive cruise control).

\subsection{Literature search approach}

To identify relevant studies for the inclusion into the DSS, a systematic scoping review was conducted for each item in the taxonomy. The aim of this approach is to represent the body of literature in a scientific way. While the criteria applied differed between research fields, there was a schematic approach followed for each review, consisting of initial search, screening, identifying additional papers, and prioritizing papers for coding (Martensen \& Lassarre, 2018).

For the initial search, several relevant literature databases were searched, e.g., Scopus, Medline, and Google scholar based on well-defined logical strings of keywords. (See Table 3, as an example). The keywords as well as the resulting number of studies were documented.

Table 3: An Example of search terms (for the risk factor “fatigue”)

\begin{tabular}{|c|c|}
\hline Fatigue & $\begin{array}{l}\text { “fatigue*” OR “Sleep*” OR “Tired*” OR “drowsy” } \\
\text { OR “drowsiness” OR “alert*” OR “monotony” OR } \\
\text { "time on task” }\end{array}$ \\
\hline \multicolumn{2}{|r|}{ AND } \\
\hline Road Safety & $\begin{array}{l}\text { “road safety” OR “driv*” OR “road” OR “transport” } \\
\text { OR “crash” OR “accident” OR “incident” OR } \\
\text { “traffic” OR “collision” OR ”traffic safety” OR } \\
\text { “risk” OR “measure OR “Road Casualties” OR } \\
\text { "Road Fatalities” }\end{array}$ \\
\hline
\end{tabular}

The next step consisted of screening the potentially relevant studies to assess their eligibility for further analysis. Generally, only studies with quantitative results were coded for repository. Important qualitative results were, however, included in the Synopses (see Section 2.4). Moreover topic-specific inclusion and exclusion criteria were applied and documented. This was done first on the basis of the abstract, then on the basis of the full paper. If few relevant papers had been retrieved, the reference lists of the selected papers were examined to identify any additional relevant papers.

To prioritize, a number of rules were given: If there was a meta-analysis available already, it (the most recent one) was used as the basis, and completed with additional studies published after, and consequently not included in that meta-analysis. Studies included in a meta-analysis were not included individually. If there were too many other papers, they were listed in descending order of importance for the road safety DSS, based on outcome variables, transferability, recent publication date, language and source. Note that these criteria were applied depending on how many studies were available and the field of research. Papers that evaluated measures and risks in terms of observed crashes were considered more relevant than those based on observed road safety behavior (e.g. speeding), which again were considered more relevant than studies that had other indicator variables as outcomes (e.g., self-reported behavior, driving simulator data, simulated crash data, etc.). SafetyCube is focused on Europe, therefore prioritizing European studies above US/Australian/Canadian studies, which are prioritized above studies from other countries. Other criteria were publication date (recent studies before older studies, though older studies of particular relevance were included), language (papers in English before papers in other languages), and source (peer reviewed papers before non-peer reviewed papers).

\subsection{Coding studies}


One of the main objectives of the SafetyCube project is to create a repository of estimates of risk factors and safety effects. In contrast to all existing repositories, SafetyCube departs from the perspective of risk factors which makes the type of studies included into the repository even more diverse.

The collected studies investigated the effect on different outcome variables: crash-counts, simulated crash data, injury severity, on-road driving, driving in a simulator, crash simulations, and so on. They employed a large variety of research designs: before-after studies, cross-sectional designs, case-control, induced exposure, timeseries; and statistical methods: simple comparisons of counts or means, different types of regression analyses, empirical Bayes, hazard rate, to name just a few. The enormous differences between studies constitute a big challenge for the creation of a joint database. The structure is general enough to allow coding different kinds of safety- or risk effects and flexible enough to capture all important details of different types of studies. For each study, the template therefore includes general information of the sampling frame and study conditions (e.g. road user types, severity of crashes, road types included), but also allows for the inclusion of conditions that are relevant to the specific area only (e.g. the differentiation between different injury types or details of the roadway design). Furthermore, for each estimated effect the following specifications were registered:

- what was compared to what

- $\quad$ analysis method/model

- $\quad$ measure of effect (often odds ratio but also many other less used measures of effect)

- $\quad$ statistical results (standard error, confidence interval)

- conclusion (significant effect on road-safety or not).

The selected studies are individually coded in an Excel coding template. The coding template consists of several sheets, requiring the researcher to provide information, mostly in predefined categories. On the basis of the study features coded, a result table shapes itself in which the results for all conditions can be entered.

Another important issue is the quality of research results. Possible biases of a particular study are coded with an indication how severe this possibility is believed to be. To this end, common biases for the major research designs are described and included into the coding template, so that these (or other) problems can be flagged if necessary.

Table 4: Overview of potential sources of bias per study design (example)

\begin{tabular}{lcl}
\hline Study design & Most common estimator of effect & Potential sources of bias \\
\hline Experiments & Odds ratio & Pre-trial non-equivalence \\
& & Differential attrition \\
& & Diffusion of treatment \\
& Unintended side-effects \\
\hline Before-after studies & Odds ratio & Regression to the mean \\
& & Long term trends \\
& Changes in traffic volume \\
& Co-incident events \\
& Use of several measures \\
& Accident migration \\
\hline
\end{tabular}

Finally, the researcher included a brief verbal summary of the study, including the main findings, as well as an assessment of their reliability and usefulness, given the study design and potential biases.

\subsection{Summarizing studies}

After having coded all selected studies, the researchers analyzed the results. Three ways had been defined to analyze and summarize the results, in a decreasing order of priority:

- Meta-analysis, if there is a sufficiently large number of studies that are comparable in terms of both their scientific design features and the type of results they produced. A meta-analysis combines the numerical results of multiple studies and yields a weighted average from the results of the individual studies [7].

- Vote-count analysis, if a meta-analysis is not possible due to large differences between studies, but if there is a sufficient number of studies. A vote-count analysis compares the share of studies that showed a positive effect, no effect, or a negative effect. 
- $\quad$ Review-type analysis, if the number of studies is small or if the studies are so heterogeneous that a votecount analysis is not meaningful. In a review-type analysis the results are summarized in a more qualitative way, generally including a table of study descriptions (e.g. sample, method, outcome), the observed effects and their interpretation.

In each type of summarizing analysis attention was dedicated towards the identification of modifying conditions (e.g. a counter-measure that works in urban, but not in rural settings or a risk-factor that is more dangerous for novice drivers). In meta- or vote count analyses this was addressed by sub-group analyses. In this context, transferability of the results is also discussed, giving an indication of situational characteristics that can impair the validity of the reported estimates for another implementation of a particular countermeasure (Martensen \& Lassarre, 2018).

For each of the studied risk factors and measures, a colour code was based on the results of the (majority of) the studies'outcomes to indicate the overall conclusion about the effect. Each colour code is supported by a short statement of two to three sentences.

Table 5: Colour code for risk factors and countermeasures

\begin{tabular}{|l|l|l|l|}
\hline \multicolumn{2}{|c|}{ Risk factor } & \multicolumn{2}{c|}{ Countermeasure } \\
\hline Red & $\begin{array}{l}\text { Results consistently show an increased } \\
\text { risk when exposed to the risk factor } \\
\text { concerned. }\end{array}$ & Green & $\begin{array}{l}\text { Results consistently show that the } \\
\text { countermeasure reduces road safety risk. }\end{array}$ \\
\hline Yellow & $\begin{array}{l}\text { There is some indication that exposure } \\
\text { to the risk factor increases risk, but } \\
\text { results are not consistent. }\end{array}$ & $\begin{array}{l}\text { Light } \\
\text { green }\end{array}$ & $\begin{array}{l}\text { There is some indication that the counter } \\
\text { measure reduces road safety risk, but } \\
\text { results are not consistent. }\end{array}$ \\
\hline Grey & $\begin{array}{l}\text { No conclusion possible because of few } \\
\text { studies with inconsistent results, or few } \\
\text { studies with weak indicators, or an } \\
\text { equal amount of studies with no (or } \\
\text { opposite) effect. }\end{array}$ & Grey & $\begin{array}{l}\text { No conclusion possible because of few } \\
\text { studies with inconsistent results, or few } \\
\text { studies with weak indicators, or an equal } \\
\text { amount of studies with no (or opposite) } \\
\text { effect. }\end{array}$ \\
\hline Green & $\begin{array}{l}\text { Results consistently show that exposure } \\
\text { to the presumed risk factor does not } \\
\text { increase risk. }\end{array}$ & Red & $\begin{array}{l}\text { Results consistently show that this } \\
\text { measure does NOT reduce road safety } \\
\text { risk and may even increase it. }\end{array}$ \\
\hline
\end{tabular}

Finally, for each risk factor or road safety measure with sufficient reference documents (typically more than 5 publications), a synopsis has been compiled. The synopsis provides a synthesis of the findings for a specific risk factor or road safety measure, including both quantitative information from the coded studies and more qualitative information from previous review studies. The synopsis aims to complement other outputs of the DSS like lists of available studies and direct access to the results of individual studies (see Section 7).

Each synopsis consists of three parts:

- Summary: In maximum two pages, the summary very briefly reports some background of the topic concerned, and the main results and conclusions based on the analysis.

- Scientific overview: In approximately four to five pages, the scientific overview describes the essence of the way the reported effects have been estimated, including a full analysis of the methods and results, and its transferability conditions in order to give the user all the necessary information to understand the results and assess their validity.

- Supporting documentation: The supporting documentation gives a more elaborate description of the literature search strategy, as well as the details of the study designs and methods, the analysis method(s) and the analysis results. Here, also a full list of coded studies and their main features is provided.

\subsection{Economic efficiency evaluation}

The countermeasures for which the analysis of safety effects has resulted in an estimated reduction of crash occurrence are submitted to an economic efficiency evaluation. A tool for Economic Efficiency Evaluation (E3calculator) of road safety counter measures is built. This tool allows to combine the information about the effectiveness of a measure (i.e. the percentage of crashes or casualties prevented) with the costs of these measures.

In a cost-benefit analysis the benefits are weighed against the costs. To be able to consider different kinds of benefits (e.g. prevented crashes or casualties of different severities) they all have to be converted to a monetary 
value (Wijnen \& Weseman, 2004; Hakkert, Wesemann, \& de Blaeij, 2009; Elvik, 2001; Martensen, Van den Berghe, Wijnen, Weijermars, Carnis, Elvik, 2016; Martensen et al. 2018). This was enabled by the collection of crash-cost estimates from European countries.

\section{Crash-cost estimation}

In a survey to experts in 31 European countries, the estimated costs from road-safety crashes were collected as a basis to calculate the cost per crash. First an overview was presented of the components that should be included in crash cost estimates and how each cost component should be determined according to the international guidelines and best practices (Alfaro, Chapuis, Fabre, 1994; ECMT, 1998; Bickel et al., 2006). The component logic was applied to costs per crash and costs per casualty. Second, information on costs of crashes and costs of casualties was collected by means of a survey among all EU countries. Four severity levels were differentiated: fatal, serious injuries, slight injuries, and damage only (with the last category available for crashcosts but not casualty costs). Third, for some countries not all information is available or costs are not calculated according to the international guidelines. In those cases, additionally to the country's own estimates (if available) comparable estimates according to the common methodology were provided by means of value transfer. In that way, EU standard values for the costs of crashes and casualties were provided as well (Wijnen, et al.; 2017).

\section{E3-calculator}

The E3 calculator brings together the results from the effectiveness studies - the number of crashes or casualties that a measure is expected to prevent - and the estimated costs of these crashes. Together these form the benefits of a road safety countermeasure, which are contrasted with the costs for the implementation and maintenance of the countermeasure.

As input to the calculator for the Economic Efficiency Evaluation, the following is needed

- Country for which evaluation is conducted

- Costs of countermeasure

o Initial costs

o Annual costs

o Source country \& year

- $\quad$ Number of crashes / casualties prevented (for each level of severity)

o Target crashes of countermeasure

o \% reduction

- $\quad$ Time horizon of a measure

On the basis of this input, the costs of the prevented crashes are based on the crash- or casualty costs for the analysis country) and the costs of the countermeasure are corrected for inflation as well as price-level differences by means of value transfer. The calculator adds for each year within the time horizon the present value of all costs and benefits. For more details see Martensen et al. (2018). Two criteria are calculated from this: (1) The total benefits are divided by the total costs to form the benefit to cost ratio and (2) the total costs are subtracted from the total benefits to calculate the net present value.

If no costs for the countermeasure are available, the calculator still produces the total benefits, which is equal to the break-even point for the countermeasure under analysis. This is the maximum cost for the counter-measure to still be considered economically efficient.

\subsection{Quality assurance}

Strict quality assurance procedures were put in place: 1 . Comprehensive guidelines, supported by workshops, webinars, Q\&A sessions, and a help desk assisted the expert SafetyCube researchers with their work. 2. The selection and coding of studies, as well as the analyses and synopses of the findings were subjected to an internal peer review. 3. A small pool of independent experts checked both the information about individual coded studies and the overall contents of the synopses, applying a set of predefined quality criteria and procedures. One expert specifically looked at consistency within and between synopses. 4. All synopses went through a language check by a native English speaker.

\section{DSS webtool}

The DSS consists of the backend (relational database), the front end (website) and the way they integrate (queries). Both study-templates and synopses are stored in a relational database, which serves as the back-end of 
the DSS. Front-end DSS results are retrieved through queries on the back-end database (DSS search engine) (Yannis \& Papadimitriou, 2018).

In order to optimize and facilitate the use of the DSS, the design of the front end system was based on the following principles:

- $\quad$ Linked search and linked results: the user should be able to search a road safety risk alone or through the measures, search a measure alone or through the risks, search for risks and measures related to specific road user groups or accident categories, and so on.

- $\quad$ Fine level of detail: the user should be able to refine the search and filter the results with many parameters among those found in the database (e.g. road types, road user groups, countries etc.)

- Flexibility: the user should be able to continuously adjust the search according to the results

- Transparency: the process is fully documented and the user should be able to access background information at any stage (links, etc.)

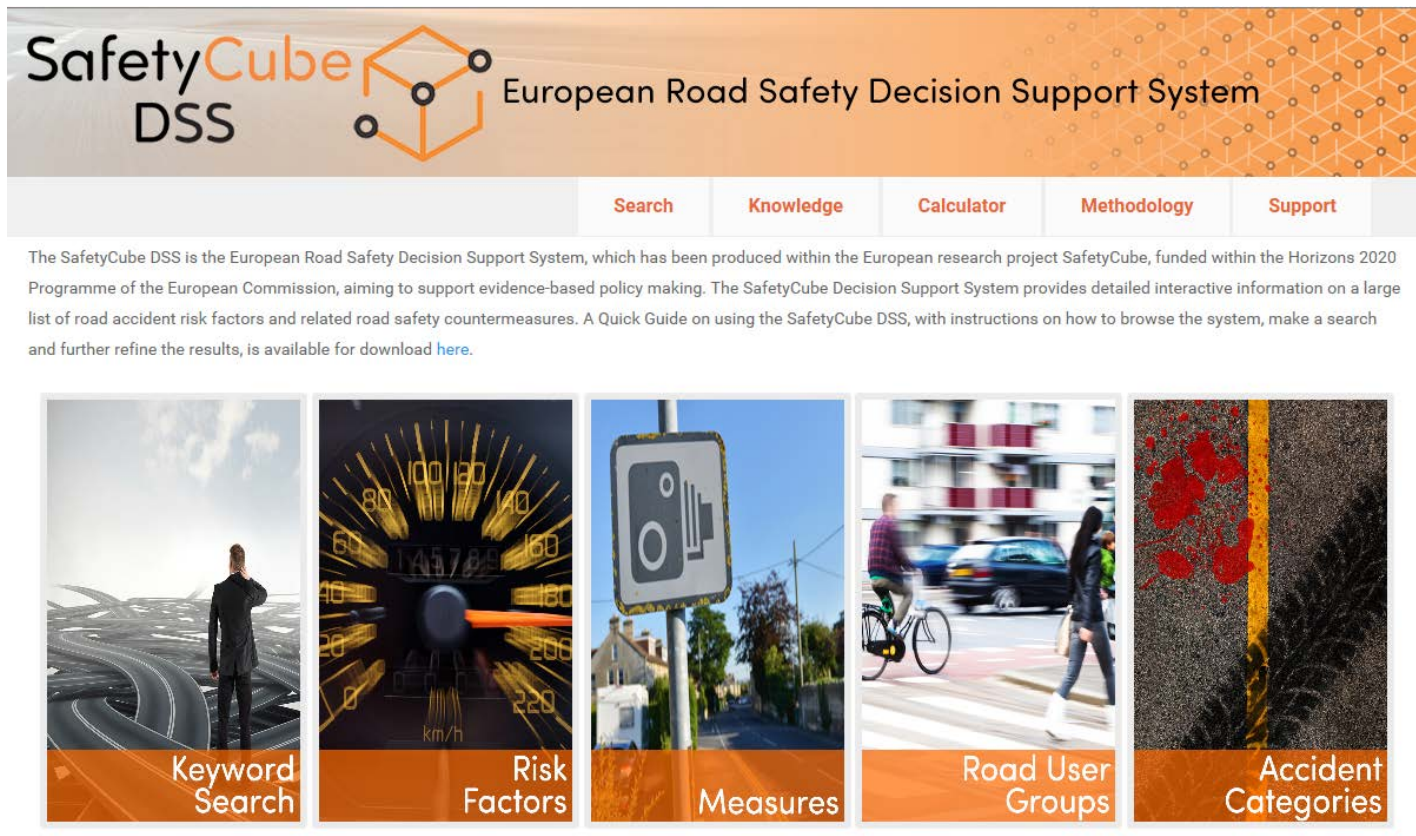

Figure 1: Main menu and entry points of the DSS.

The SafetyCube DSS Search is structured in three operational levels (with Level 0 being the system Home Page), as shown in Figure 2: Level 1 includes the Search Pages, Level 2 includes the Results Pages and Level 3 includes the Individual study pages. These are further described below (for a full description, see Yannis \& Papadimitriou, 2018).

The Search Pages include a dynamic part and a static part (see Figure 1). The dynamic part concerns:

- The Search tab allows the user to query the DSS backend database and retrieve results for risk factors or measures, through five entry points (keywords, risk factors, measures, road user groups, accident scenarios), all leading to a Results page. Moreover, links between risks and measures are implemented in the results pages. The results pages include all the DSS outputs in terms of coded studies, summaries etc., and a selection of one of these outputs will lead to the Individual study page with detailed information on the specific study.

- The Calculator, a one-page web application which allows the user to retrieve one of the SafetyCube examples of cost-benefit analysis, edit it with own values or perform his/her own cost-benefit analysis of a road safety measure.

The static part includes additional one-level pages with supporting documentation, text and links as follows:

- The Knowledge tab, which compiles the SafetyCube key documents as a knowledge library. This includes the Synopses of all risk factors and measures (which may be filtered or sorted per topic), Accident Scenarios fact-sheets with information and data on the most important crash 
configurations, and documentation regarding serious injuries (i.e. estimating the number of serious injuries, estimating the costs and impacts of serious injuries, risk factors related to serious injuries)

- The Methodology tab, which includes key background information and documents on the SafetyCube methodology and related disclaimers, a glossary of terms used and information on the quality assurance procedures implemented.

- $\quad$ The Support tab: includes contact information, the guide to DSS users, possibility to send feedback or questions, and useful links to other systems.

More specifically, the Search Tab (Level 1) consists of the specific search that the user wishes to carry out on the basis of the five entry points mentioned above. The users will be able to select one of these five entry points depending on the type of search that they wish to conduct. More specifically, the text search allows the users to enter a key-word (selected among the key-words of the studies included in the database), the road safety risk factors/measures entry points allows them to seek specific risk factors or measures from the SafetyCube taxonomies respectively. As a shortcut to the results concerning crash risks or measures specifically related to a road user group, one may enter via the road user groups query (7 groups e.g., pedestrian or powered twowheeler) or via the accident scenario query (8 categories e.g. head-on collision or rear-end collision). The system will always respond with a subset of risk and measure taxonomies that are relevant in relation to the selected road user group or accident scenario and the resulting studies will be only those that are related to the road user group or accident scenario in question.

It is important to highlight that the entire search component is based on two interlinked pillars, risk factors and road safety measures. In fact, all entry points at Level 1 eventually lead to a selection of risk factors or measures of interest, and only by selecting one does the user retrieve the results at Level 2.

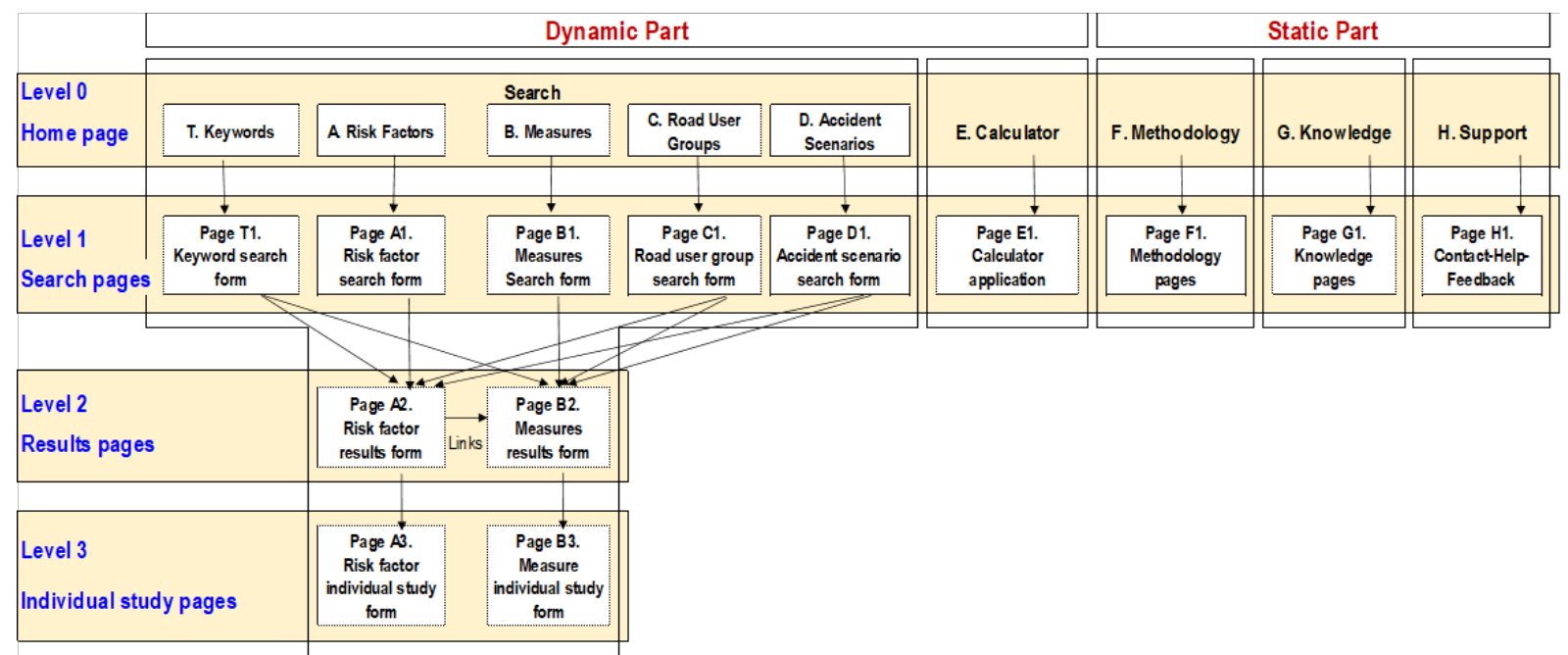

Figure 2: Overview of the Main Menu and entry points of the DSS.

In the Results pages (Level 2), upon selecting an entry on one of the above lists (risk factors or measures), the main results will appear. The results consist of:

- $\quad$ Short introductory texts and the colour code(s), describing the risks or the effectiveness of measures.

- Links to one or more available SafetyCube synopses on the issue (pdf link button(s) next to the colour code).

- A table listing the available meta-analyses and other coded studies in the SafetyCube database together with their main characteristics such as design, country, and year of publication. Selecting a study from the Table will lead the user to the individual study page.

- $\quad$ Depending on the selected topic, adaptive search filters are available on the left side of the results page. Filters include: keyword, specific risk factor (corresponding to the most detailed taxonomy level), road user group, road type, country. The keyword filter appears only when entering from the "keyword" or "road user group" entry point, and allows the user to "un-filter" the results and obtain all the studies related to the risk factor or measure (and not only those related to the keyword or road user group).

- A button which links to related measures (if the results page is in the risks domain) or to related risk factors (if the results page is in the measures domain). 
- If Economic Efficiency Evaluations (E3) are available for a measure, a link to the pdf. Document containing the results is displayed, allowing the user to access all the calculations and background information.

Finally, the user may select a specific study from the results page, and have the individual study results (provided in Level 3), including the study abstract as available in the original publication, the related URL, and a table of all risk / measure safety effects available in the study containing test and reference condition (e.g. helmet vs. not helmet), type of outcome (e.g. injury severity), type of estimate (e.g. odds ratio), statistical significance and confidence intervals.

\section{Discussion}

The DSS was developed as a tool for developing evidence based road safety policies. It allows users to understand risk factors and select the most appropriate and cost-effective measures. The DSS allows those developing road safety policies to access information from the whole road traffic system - road users, infrastructure, vehicles, post-impact care - in one place as well as indicating the relationship between risks and measures in the linking system. The information provided on the evidence for risk and effectiveness of measures is based on a rigorous standardized methodology that prioritizes high quality peer-review publications. Such information is often not publicly accessible under 'open access' criteria so the DSS allows users to access scientific evidence without needing access to the original source documentation. The synopses also provide information in a way that can be understood by users of a wide range of backgrounds - for those new to the road safety field as well as those experienced. In addition the E3 calculator provides a tool to assess the cost effectiveness of measures and to compare the costs of measures in a standardized way (although using the E3 tool, however, does require a good knowledge of its development and the user guidelines and it is helpful for the user to have an understanding of cost benefit analyses).

Overall, it is the only road safety DSS containing comprehensive and linked information both on crash risks and measures so that users are directed from problems to solutions on a user-friendly graphical interface. It locates both risks and measures in robust taxonomies, mapping the whole road safety domain, across the fields of human behavior, infrastructure, vehicles and post-impact care. This allows users with various backgrounds to benefit from the vast knowledge contained in the system by casting scientific evidence on every risk and every measure (or groups thereof) into comprehensive synopses, reachable through different entry points.

The DSS however, as with all tools, has some limitations. One might compare it to the most famous database of studies and reviews - the Cochrane Database of Systematic Reviews (CDSR, www.cochrane.org). Although the basic idea behind the DSS is the same, the approach to study quality is different. The Cochrane protocol (Higgins \& Green, 2011) is very rigid in terms of study-inclusion criteria and focuses mainly on controlled randomized trial experiments. This is indeed the ideal methodology to be sure about the causal relation between a treatment (e.g., medicine, therapy or a road safety countermeasure) and its intended effects. Road safety crashes, the "disease" addressed in the DSS are, however, very violent events and fortunately also quite rare. This makes them unsuited to be observed in randomized control trial experiments due to ethical as well as practical constraints. The philosophy behind the DSS is therefore to collect the best of the available evidence. For safety evaluation of roadway sites, Empirical Bayes before-after studies are considered to be the golden standard (Persaud \& Lyon, 2007; Elvik, 2012), but many, even scholarly published, studies do not fit all the established criteria (such as correcting for regression to the mean as well as correcting for trend effects and for changes in traffic volume), often due to data constraints. For safety evaluation of other safety measures, the best available knowledge comes in the ideal case from 'multiple studies employing multiple methods, all leading to the same conclusions' (Shinar, 2007). However, there are not many examples of this. All in all this means that, even after a few decades of research, the scientific knowledge base for some topics is still quite limited. For example, studies of emerging technologies, like ECall (automatic crash notification systems), containing subjective assessments of small datasets (Sirola, Luoma, Shirokoff, Salo, \& Karkola, 2009; Olin, Strandroth, \& Lie, 2014; Ponte, Ryan, \& Anderso, 2016) were reviewed and coded for the DSS. While statistical analyses were limited in these reports, the estimates of the measure's effects are important to convey to road safety stakeholders. Our approach has been to include the best possible information and to take the limitations of the studies into account when describing the state of the knowledge in the synopses summarizing the results. For the future, it would be hoped to gradually teach the research community which studies are likely to produce unbiased results and how to report results in a conclusive way. In this way the evidence for topics with few highquality studies could be improved. Apart from the availability of good research, at the time being, the DSS does 
not have detailed information on all possible road safety topics. The scope of the SafetyCube project has allowed to synthesize the knowledge on more than 200 topics (risks \& measures) and it is hoped that more topics can be added to the DSS in the future.

Moreover, a Safe System approach seeks that no human is killed or seriously injured. However, scientific studies more commonly consider the impact of risks and measures on police reported crash frequency or crash severity. The DSS considers serious injury as a specific topic and includes numerous studies with impacts on injury severity, yet is bounded by available studies. Most evaluation studies are based on police reported crashes or casualties and it is known that non-fatal crashes are underreported by the police and that police reporting rates differ between crash types (e.g. Amoros, Martin, \& Laumon, 2006; Elvik \& Mysen, 1999; Janstrup, Kaplan, Hels, Lauritsen, \& Prato, 2016). Therefore, impacts on serious injuries should be further analyzed for the strengthening of the safe systems perspective.

The DSS focused on Europe which could pose limitations on the transferability of information to other regions/countries. Although studies conducted in Europe were priorities, studies from other countries such as the USA, Australia and Japan were included. In addition, consideration of the transferability of knowledge to other countries/contexts was given within the synopses. Another potential limitation to the use of the DSS is the challenge of keeping it up-to-date over time. Funding is actively being sort so that the contents of the DSS can be updated and complemented in the years to come. Last but not least, the current DSS content contains mainly studies of road safety measures in isolation. A challenge to the research community is to depart from traditional research silos and evaluate measures in combination. Whenever study results of joint effects of measures were available, this was presented in the synopses. However, typically this concerned effects of measures from the same domain (e.g. enforcement and awareness campaigns, Philips, Ulleberg, \& Vaa, (2011). The novel SafetyCube approach to linking risks and measures demonstrates new potential areas where measures can be combined. It is hoped that future research will use these links and evaluate a combined measures approach.

\section{Conclusion}

More than 1500 studies evaluating risks or measures have been analysed with more than 7000 estimates of risks or measure effects coded. They have been summarised in 211 synopses $[18,19,20,21,22,23,24]$ and more than 35 benefit-cost analyses [25,26].

The SafetyCube DSS (Decision Support System) is available at the following URL: http://www.roadsafetydss.eu. Its pilot operation started early 2017; since then the system has been updated continuously until April 2018 (end of the SafetyCube project) and beyond.

The DSS is intended to become a major source of information for industry, policy makers and the wider road safety community; it incorporates the knowledge base of accident causation, risks and measures developed in the project and the underlying methodological systems. The content of the DSS is holistic in nature as it includes risks and measures in the road user, infrastructure, vehicle, post impact care domains. It provides information in an accessible way to a wide variety of potential users. The DSS, therefore, has a great potential to further support evidence-based decision making at local, regional, national and international level, aiming to fill in the current gap of comparable measures effectiveness evaluation across Europe and worldwide.

\section{References}

Alfaro, J. L., Chapuis, M., Fabre, F. (Eds.) (1994). Socio-economic cost of road accidents: final report of action COST 313. Commission of the European Community, Brussels.

Amoros, E., Martin, J.-L., \& Laumon, B. (2006). Under-reporting of road crash casualties in France. Accident Analysis \& Prevention, 38(4), 627-635. doi:https://doi.org/10.1016/j.aap.2005.11.006

Bickel, P.; Friedrich, R.; Burgress, A; Fagiani, P.; Hunt, A.; De Jong, G.; Laird, J.; Lieb, C.; Lindberg, G., Mackie, P., Navrud, S.; Odgaard, T; Ricci, A.; Shires, J., Tavasszy, L. (2006). Proposal for harmonised guidelines. EU project HEATCO Deliverable 5. University of Stuttgart, Stuttgart.

CMF Clearinghouse. Crash Modification Factor Clearinghouse. Federal Highway Administration, US Department of Transportation. http://www.cmfclearinghouse.org/index.cfm (accessed February, 2018)

Daniels S., Papadimitriou E. (Eds) (2017). Economic evaluation of infrastructure related measures, Deliverable 5.3 of the H2020 project SafetyCube.

Daniels, S., Aigner-Breuss, E., Kaiser, S., Goldenbeld, C., Katrakazas, C., Schoeters, A., Ziakopoulos, Usami, D.S., Bauer, R., Papadimitriou, E., Weijermars, W., Rodriguez Palmeiro, A. \& Talbot (2017). Economic evaluation of road user related measures. Deliverable 4.3 of the H2020 project SafetyCube.

ECMT (1998). Efficient transport for Europe; Policies for internalisation of external costs. Organisation for Economic Cooperation and Development OECD, Paris.

Elvik R. (2004). To what extent can theory account for the findings of road safety evaluation studies? Accident Analysis and Prevention 36 (2004) 841-849. 
Elvik, R. 2001. Cost-benefit analysis of road safety measures: applicability and controversies. Accident Analysis and Prevention, 33, 9-17.

Elvik, R., \& Mysen, A. (1999). Incomplete Accident Reporting: Meta-Analysis of Studies Made in 13 Countries. Transportation Research Record: Journal of the Transportation Research Board, 1665, 133-140. doi:10.3141/166518

Elvik, R., (2012). Analytic choices in road safety evaluation: Exploring second-best approaches. Accid. Anal. Prev. 45, 173179. doi:10.1016/J.AAP.2011.12.006

Elvik, R., Hoye, A.; Vaa, T., Sorensen, M. (2009). The handbook of road safety measures. 2nd Edition. Emerald, Bingley, UK.

Filtness A. \& Papadimitriou E. (Eds) (2016), Identification of Infrastructure Related Risk Factors, Deliverable 5.1 of the H2020 project SafetyCube.

Filtness A., Thomas P., Talbot R., Quigley C., Papadimitriou E., Yannis G., Theofilatos A., Aigner-Breuss E., Kaiser S., Machata K., Weijermars W., Van Schagen I., Hermitte T (2016), The application of systems approach for road safety policy making, Deliverable 8.1 of the H2020 project SafetyCube.

Goodwin, A., Thomas, L., Kirley, B., Hall, W., O’Brien, N., \& Hill, K. (2015, November). Countermeasures that work: A highway safety countermeasure guide for State highway safety offices, Eighth edition. (Report No. DOT HS 812 202). Washington, DC: National Highway Traffic Safety Administration.

Haddon W Jr. (1980). Advances in the epidemiology of injuries as a basis for public policy. Public Health Rep. 95 (5): $411-$ 21.

Hakkert, S. \& Wesemann, P. eds (2004). The use of efficiency assessment tools. Solutions to barriers. ROSEBUD Report Nr. 5.

Higgins JPT, Green S (editors). Cochrane Handbook for Systematic Reviews of Interventions Version 5.1.0 [updated March 2011]. The Cochrane Collaboration, 2011. Available from www.handbook.cochrane.org.

Janstrup, K. H., Kaplan, S., Hels, T., Lauritsen, J., \& Prato, C. G. (2016). Understanding Traffic Crash Under-Reporting: Linking Police and Medical Records to Individual and Crash Characteristics. Traffic Injury Prevention, 17(6), pp 580-584. Retrieved from http://dx.doi.org/10.1080/15389588.2015.1128533.

Machata K., Papadimitriou E., Soteropoulos A., Stadlbauer S. (Eds) (2017), Identification of safety effects of infrastructure related measures, Deliverable 5.2 of the H2020 project SafetyCube.

Martensen, H, Van den Bergh, W, Wijnen, W., Weijermars, W., Carnis, L, Elvik, R. (2016), Preliminary guidelines for priority setting between measures, Deliverable Number 3.4 of the H2020 project SafetyCube.

Martensen, H., and Lassarre S. Eds (2017), Methodological framework for the evaluation of road safety measures, Deliverable 3.3 of the H2020 project SafetyCube.

Martensen, H., Daniels, S; Van den Berghe W., Wijnen, W., Weijermars, W., Carnis, L., Saadé, J., Elvik, R. (2018) , Guidelines for priority setting between measures with practical examples, Deliverable Number 3.5 of the H2020 project SafetyCube.

Ohlin, M.;Strandroth, J.;Lie, A.(2014). Potential safety benefits of Automatic Collision Notification - A case by case analysis, 21st World Congress on Intelligent Transport Systems, ITSWC 2014:

Persaud, B., Lyon, C., (2007). Empirical Bayes before-after safety studies: Lessons learned from two decades of experience and future directions. Accid. Anal. Prev. 39, 546-555. doi:10.1016/J.AAP.2006.09.009

Phillips, R., Ulleberg, P. \& Vaa, T. (2011): Meta-analysis of the effect of road safety campaigns on accidents. Accident Analysis and Prevention, 43, 1204-1218.

Ponte, G.; Ryan, G. A.;Anderson, R. W. G.(2016). An estimate of the effectiveness of an in-vehicle automatic collision notification system in reducing road crash fatalities in South Australia, Traffic Injury Prevention, www.tandfonline.com/doi/full/10.1080/15389588.2015.1060556

Reed, S., Weijermars, W, et al. (2017), Identification of key risk factors related to serious road injuries and their health impacts, Deliverable 7.4 of the H2020 project SafetyCube.

Shinar, D.,( 2007). Traffic Safety and Human Behaviour. Elsevier, Oxford.

Sihvola, N.; Luoma, J.; Schirokoff, A.; Salo, J.; Karkola, K. (2009). In-depth evaluation of the effects of an automatic emergency call system on road fatalities, European Transport Research Review; 2009:1-3

Talbot, R., Aigner-Breuss, E., Kaiser, S., Alfonsi, R., Braun, E., Eichhorn, A., Etienne, V., Filtness, A., Gabaude, C., Goldenbeld, C., Hay, M., Jänsch, M., Leblud, J., Leskovšek, B., Paire- Ficout, L., Papadimitriou, E., Pilgerstorfer, M., Rußwurm, K., Sandin, J., Soteropoulos, A., Strand, N., Theofilatos, A., Van Schagen, I., Yannis, G., Ziakopoulos, A. (2016), Identification of Road User Related Risk Factors, Deliverable 4.1 of the H2020 project SafetyCube.

Theofilatos, A., Aigner-Breuss, E., Kaiser, S., Alfonsi, R., Braun, E., Eichhorn, A. et al. (2017), Identification and Safety Effects of Road User Related Measures. Deliverable 4.2 of the H2020 project SafetyCube.

Wijnen, W., Weijermars, W., Vanden Berghe, E., Schoeters, A., Bauer, R., Carnis, L., Elvik, R., Theofilatos, A., Filtness, A., Reed, S., Perez, C. and Martensen, H. (2017), Crash cost estimates for European countries, D3.2 of the H2020 project SafetyCube.

Wijnen, W., Wesemann, P. \& de Blaeij (2009). Valuation of road safety effects in cost-benefit analysis. Evaluation and Program Planning, 32, pp. 326-331.

Yannis G., Papadimitriou E. (Eds) (2018), The European Road Safety Decision Support System - A clearinghouse of road safety risks and measures, Deliverable 8.3 of the H2020 project SafetyCube. 